\title{
3D-4D Ultrasound in the First Trimester of Pregnancy
}

\author{
Carolina Axiana, Maria Angelica Zoppi, Rosa Maria Ibba, Marcella Floris, Fabiola Manca, Giovanni Monni \\ Department of Obstetrics and Gynecology, Prenatal and Preimplantation Genetic Diagnosis \\ Fetal Therapy, Ospedale Microcitemico, Cagliari, Italy
}

Correspondence: Dr Giovanni Monni, Department of Obstetrics and Gynecology, Prenatal Diagnosis, Fetal Therapy, Ospedale Microcitemico, via Jenner, 09121 Cagliari, Italy

Tel. +39070 505306 +39070 6095546-7, Fax +39070 6095514, e-mail: prenatalmonni@tiscali.it

\begin{abstract}
Three-dimensional ultrasound (3DUS) imaging is a new technique that allows imaging from volume sonographic data rather than conventional planar data. The volume data can be viewed as a three-dimensional object and displayed in multiplanar mode in three orthogonal planes or in various modes of rendering that can be selected singularly or combined to optimize the visualization of soft tissues, bony structures or vessels.

The 4D imaging has introduced a temporal dimension to the 3D volumetric scansion, associating movement to representation of volumes.

3DUS provides the ability to examine the embryo and the fetus at the first trimester in any arbitrary plane. Various studies have reported a better visualization of the structures in normal fetuses as well as malformed ones, regarding selected cases of facial structures, cranium, abdominal wall and limbs.

3DUS technique is also being considered for measurement of nuchal translucency and the evaluation of the nasal bones.

Several reports exist on the application of 3D-4D techniques regarding conjoined twins.

4DUS allows the evaluation of fetal motorial and behavioral patterns.

3D-4D techniques are seen as a powerful complement to conventional ultrasound, but not a substitution to it.
\end{abstract}

Key words: 3D ultrasound/4D ultrasound, fetus, first trimester pregnancy.

\section{INTRODUCTION}

The application of ultrasound technology for the study of the fetus has with no doubt revolutionized the obstetrics field. Before the introduction of the sonogram diagnosis, we had very little information about the health and development of the fetus. At the beginning of the 1970s two dimensional staticscanning ultrasound (2DUS) allowed for the first time a viewing of the fetus. Further progress was reached with the real-time-Bmode imaging at the beginning of the 1980s. Subsequently in the late 1980s beginning of the 1990s with improvement of traditional equipment, imaging has improved so much that volume and its multiplanar display was acquired (3DUS) and more recently the real time $3 \mathrm{D}$ (or 4D). ${ }^{1-3}$
The application of 3DUS in obstetric imaging is positive in showing information not immediately available with the 2DUS technique as far as fetus development and abnormalities are concerned. $^{4-5}$

Initially there have been various reports on normal fetuses as well as malformed ones, with subsequent works showing detailed images of organs or specific districts.

The data from some centers suggested that 3DUS compared to the conventional 2DUS can provide further information on the evaluations of fetal anomalies. Merz et al have examined 204 patients, and have found that the 3DUS was better in showing fetal defects in 127 patients (62\%) equivalent in 73 $(36 \%)$ and worse in $4(2 \%){ }^{4}$

Dyson and Pretorius et al, reported similar results in 63 patients with 103 anomalies: the 3DUS was positive in $53(51 \%)$ equivalent in $46(45 \%)$ and negative in $4(4 \%){ }^{6}$

Moreover single reports regarding selected cases of facial structures, cranium, abdominal wall and limbs have confirmed these data.

The improvement of imaging ultrasound through multiplanar display and rendered images allowed a better visualization of the structures with 3DUS compared to 2DUS in those cases, contributing to a more thorough understanding of anomalies by operators as well as the families. ${ }^{4-5}$

\section{TECHNICAL ASPECTS}

While with the conventional ultrasound 2D we get information referring to single planes of the structure, with 3DUS we have a volume of data with subsequent vision in multiplanar mode in three orthogonal planes (3D multiplanar display, the last plane is the coronal one that cannot be acquired directly), or in surface rendering mode with which the data of a whole volume is converted into a single image, displaying the surface of the examined structure.

The rotation of the data of the volumes in standard orientation allows a simultaneous viewing of the sagittal, transverse and coronal planes, which often gives diagnostic 
images even when the fetus is poorly positioned. Various modes of rendering can be selected, singularly or combined, to optimize the visualization of a particular organ or structure of the fetus.

Surface rendering allows the visualization of the fetus external body as well as the internal surface structure, which is particularly useful to outline the cranial vault, facial structures and limbs.

The examination of fetal limbs with surface rendering allows us to see various segments and to point out any pathologies of morphologic anomalies of the limbs. Anomalies of the fetus bony structures can be represented with the maximum transparency mode providing a solely image of the highly hyperechogenic structures that favors the structural and topographical evaluations of the anomalies. The quality of the $3 \mathrm{D}$ image depends on a process that requires an operator, who determines the outcome with his best choice of 2D image, manipulation of the images and the fictitious movements generated by the fetal and maternal movements.

Differing from static 3D, real time 4D mode is obtained through continuous volume acquisition and parallel calculation of 3D rendered images. With imaging 4D we have introduced a temporal dimension to the $3 \mathrm{D}$ volumetric scansion. This gave us not only the representation of volumes but also the association of movement. ${ }^{7,8}$

\section{FACIAL ANOMALIES}

Without doubt evaluation of facial structures is considered one of the best fields for the application of the 3DUS. 3D reconstruction of the fetus' face allows an immediate understanding and better rendering for the operators and the expectant Mother and it is better than any other image obtained with a 2D technique. Merz et $a l^{9}$ have reported cases in which 3D transvaginal ultrasound provided surprisingly well-defined facial images as early as the 9 th week of pregnancy. The 3DUS can have a role in evaluating cranial-facial malformation in early stages of the pregnancy. Moreover we navigate through slicing inside the volume and we have the possibility to rotate the volume data according to the three orthographic planes allowing the structures to be evaluated integrally and in adequate planes.

Some abnormalities have been diagnosed with 3D ultrasound. Among them facial cleft, micronathia, nasal abnormalities and eye abnormalities. ${ }^{10,11}$ Some authors have reported a 3D volume rendering of a case of frontonasal malformation within the first trimester during the 12th week of pregnancy. The direct visualization of the fetal face through 3D surface rendering allowed a detailed description of the characteristics of the frontonasal malformation and confirmed by fetoscopy and autoptic examination.

The combination of surface and maximum intensity modes showed that the frontal bones were largely separated, as were nasal bones. This pathology, considered a median cleft syndrome, is the result of the anomalous development of the nasal capsule (the cartilage involucres containing nasal structures) and is characterized by an ocular hyperterlorism, flat nasal bridge, rudimentary nostrils, lack of extremities in the nasal dorsum and anterior cranium bifidum occultum. ${ }^{11}$

\section{SKULL ANOMALIES}

The ossification of the cranial vault starts around the 10th week and ends between the 11th and 12th week; it is possible to suspect malformations such as acrania, exencephalia, anencephalia, using a transvaginal 2D ultrasound, but the final diagnosis is possible only after the 11 th or 12 th week. It is believed that acrania and exencephalia are precursories of anencephalia which develops as a result of a defective closure of the rostral neuropore during the 5th week of pregnancy. Its morphological abnormality is well defined and outlined by the absence of the cranial vault and the protrusion of disorganized cerebral tissue. This condition can be overlooked when depicting the optimal section is difficult. By 3D multiplanar display, depicting the correct sagittal section and coronal section, acrania and exencephaly are rather easily diagnosed. Also, by the surface mode, the abnormality of the head is shown more clearly. ${ }^{12}$ Viewing the skull sutures and the fontanelles can be difficult with 2DUS due to the natural curve of the skull. 3DUS, and in particular the use of the algorithm maximum transparency mode, is a valid support in viewing the skull sutures and the fontanelles contributing to the diagnosis of abnormalities consequent to an excessive diastase of the sutures as in the case of cranium bifidum occultum. ${ }^{11}$

\section{CENTRAL NERVOUS SYSTEM ANOMALIES}

The main objective for sonoembryology is the examination of the central nervous system (CNS) because the development stages of the brain ventricles can be confirmed by conventional 2DUS from the 7 th week onward. ${ }^{13}$ Using a method of computerized 3D image analysis Blaas et $a l^{14,15}$ were able to show the development process of the diencephalon, mesencephalon and rhombencephalon. An early diagnosis is possible in some of the more severe anomalies of the CNS like holoprosencephaly. Gonzales-Gomez et al ${ }^{16}$ have reported a alobar holoprosencephaly after only 10 weeks of pregnancy with its single ventricular cavity. Nevertheless a detailed observation of the ventricular structure is needed for an early diagnosis of the anomaly, because the degree of anomaly depends on the degree of the cleavage of the prosencephalon. Blaas and colleagues ${ }^{17}$ have reported that when holoprosencephaly is diagnosed at 9 weeks, the use of 3DUS allows additional diagnostic tomograms and the volume reconstruction improves the imaging and the understanding of such condition. 


\section{ABDOMINAL WALL DEFECTS}

All fetuses at the 8th to 9th week show an herniation of the midgut, that is visualized as a hyperechogenic mass at the base of the umbilical cord. The retraction of the midgut inside the abdominal cavity is completed around the end of the 12th week. The use of 3D multiplanar display is more accurate than the use of the 2DUS for measuring the size of the omphalocele. Chang et $\mathrm{al}^{18}$ have reported a case of omphalocele at the 14 th week of pregnancy using the 3DUS surface rendering. In gastroschisis, the herniation of the intestine is caused by a defects in the abdominal wall located on the side, usually on the right of an intact umbilical cord. The ultrasonographic diagnosis for gastroschisis is based on the demonstration of the normal localization of the umbilical cord and the hernia of the intestinal loops fluctuating freely. Congenital anomalies of the abdomen such as omphalocele and gastroschisis can be clearly evaluated through 3DUS. It is not clear if this technique gives additional information compared to 2DUS. However, the mother and her family can understand these defects more clearly and immediately with the 3DUS. The volume of defects such as omphalocele gastroschisis, can be evaluated and considered useful in the prediction of the prognostic judgment.

\section{ABNORMALITIES OF FETAL LIMBS}

3DUS provided rendering algorithms that allow a specific evaluation of the fetal bony structures. The maximum transparency mode and X-ray represent a method of rendering able to provide excellent images of hyperechogenic structures providing an accurate analysis of the majority of fetal bony structures. The buds of the upper and lower limbs can be observed as early as the 8th or 9th week. The distal segments, hands and feet, are visible at the 10th week of pregnancy. The evaluation of the long bones and the size and morphology of the distal extremities is possible starting at the 12th week. Since they are superficial structures, it is not surprising to find various reports of important anomalies related to limbs as some cases of phocomelia and sirenomelia evident at the first trimester of pregnancy with an evaluation by means of 3DUS technique ${ }^{19,20}$ (Fig. 1).

Because the 3D images are very similar to the real images of the structures, opposite to 2D images where the mental reconstruction of the three dimensional image must be done by the operator, some authors comment on the undoubted advantage of the 3DUS vs. 2DUS regarding the study of limb anomalies. ${ }^{21}$

\section{NUCHAL TRANSLUCENCY IN NASAL BONE}

An increase of nuchal translucency (NT) in the first trimester of pregnancy is a common characteristic in various chromosomic anomalies and it has been recognized as a useful indicator of

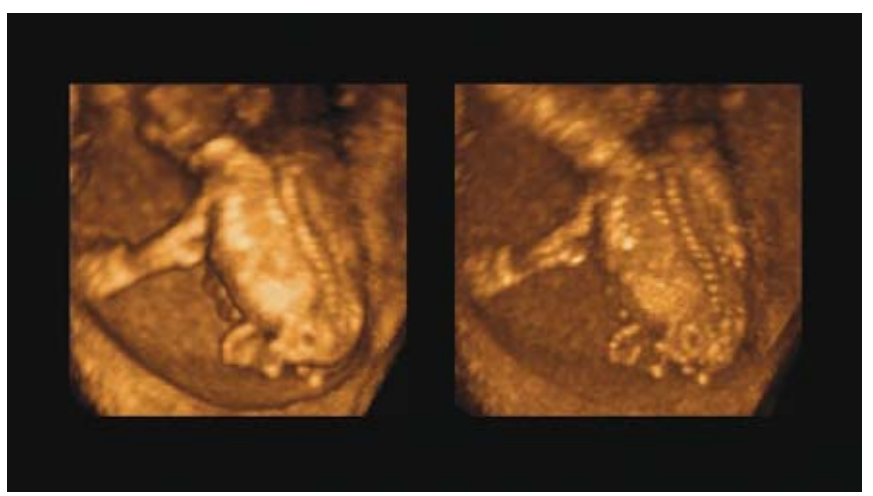

Figs 1A and B: A case of phocomelia in fetus at 12 weeks regarding lower limbs that shows only the presence of the feet in 3D surface mode $(A)$ and $3 D$ maximum transparency mode rendering $(B)$

these anomalies since the beginning of the 1990s. The conventional method for measuring NT is transabdominal 2D ultrasound, a standard technique presently used in clinical pratice. ${ }^{22}$ 3DUS is not usually employed for measuring nuchal translucency at 11-14 weeks of pregnancy. Some studies have compared the 2DUS measuring methodology of the NT versus $3 \mathrm{D}$. Kurjak et $\mathrm{l}^{23}$ have compared transvaginal 2DUS versus transvaginal 3DUS in 120 women subjected to ultrasound screening at 11-14 weeks of pregnancy. This study has shown that using the 3DUS technique, the midsagittal plane of the fetus can be visualized successfully $100 \%$ of the times versus $80 \%$ of the times with the $2 \mathrm{D}$ technique.

It also arised that 3DUS improves the accuracy in measuring the NT by allowing an appropriate midsagittal section of the fetus and a clear distinction of the nuchal region from the amniotic membrane.

Moreover because the 3D technique offers a better reproduction among operators compared to $2 \mathrm{D}$, the sensitivity of the screening is increased.

Another comparative study between 3D and 2D transvaginal US for the measurement of the nuchal translucency has been carried out by Eppel $e t \mathrm{al}^{24}$ to evaluate feasibility and the level of agreement.

It resulted that the 3D transvaginal technique offers a shorter examination time and higher success rate for measuring NT. Moreover a slight but significant underestimation of the measurement of NT was noted, but no impact in the clinical practice is known. The lack of visualization of NT through transvaginal 3DUS is fundamentally due to fetal movements while obtaining the data.

Besides the measurement of nuchal translucency, the screening for trisomy 21 during the first trimester includes the evaluation of the fetal nasal bones, as this chromosomopathy is associated to the absence of the nasal bone at 11-14 weeks of pregnancy. ${ }^{25,26}$ 
In checking the fetal nasal bone at 11-14 weeks of pregnancy, the 3DUS allows a perfect midsagittal plane of the fetal profile and the rotation of the fetal profile at any angle. However proving the presence of nasal bones in a given rebuilt section is entirely dependent on the 2D section of the starting point of the acquired volume. This is the result of a preliminary study of Rembouskos et $a l,{ }^{27}$ who have evaluated the potential role of 3DUS in overcoming major technical problems in showing the nasal bone in a perfect midsagittal plane.

Examining the fetal nasal bone when the starting 2D section is transversal or coronal, the percentage of the cases in which the nasal bone can be shown in a midsagittal plane is very low. We also have similar results in cases where the starting 2D plane is midsagittal but the angle between the ultrasound transducer and an imaginary line passing through the fetal profile is $<30^{\circ}$ or $>60^{\circ}$. It is not possible to show the presence of the nasal bone in the reconstructed image obtained through rotation of the fetal profile around its vertical axis to reach the optimal angle of $45^{\circ}$. Consequently the inability to evaluate the nasal bone through $2 \mathrm{D}$ technique due to the position of the fetus head, hyperextended or very flexed, cannot be overcome by obtaining a 3D volume. The principal value of 3DUS in evaluating the nasal bone is based on obtaining a satisfying reconstructed midsagittal section, when the starting 2D section is para-sagittal or slightly oblique.

Another recent work by Peralta $e t a l^{28}$ refers to the use of 3DUS technique in multiplanar mode. This study has highlighted the presence of a gap between the nasal bones on the axial plane fetus' face in about $20 \%$ of the fetuses, and in about $40 \%$ of these cases in the perfect midsagittal plane the nasal bone may be erroneously considered absent. 3D ultrasound can demonstrate the absence of the nasal bone in the axial plane, reducing false-positives and increasing the sensitivity. Still regarding the evaluation of nasal bones, the application of algorithm of rendering maximum transparency mode can be used instead of multiplanar mode as this better outlines the bony structures, eliminates superficial echoes and allowes to show the unilateral or bilateral absence of the nasal bone during the screening in the second trimester for the Down syndrome. The application of the same 3D technique with the rendering algorithm maximum transparency mode can highlight bony structures even in fetuses in the first trimester ${ }^{29}$ (Fig. 2).

\section{CONJOINED TWINS}

Conjoined twins are a rare anomaly in the field of monozygotic twins and are found every 50000 to 100000 births. Even though the separation of twins can be successfully performed in some selected cases, practicability and the consequent morbidity depends on the degree of condivision of the organs and vascular structures. Thus a detailed visualization of the fetuses is a fundamental element. ${ }^{30}$ Nevertheless an early and accurate

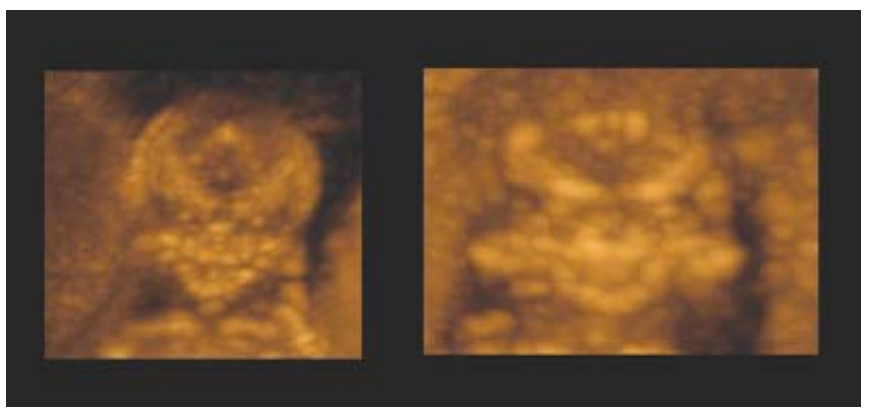

Figs $2 \mathrm{~A}$ and $\mathbf{B}$ : Fetus at 12 weeks. 3D maximum transparency mode rendering shows clearly the presence $(A)$ and the bilateral absence of both nasal bones (B).

diagnosis of conjoined twins is still a challenge because of the difficulty in seeing a complete separation of the twins, observing two spinal cords in an unusually close position, presenting embryos or fetuses in atypical shapes, or a single cardiac structure. ${ }^{31}$ An accurate prenatal diagnosis of conjoined twins can be performed through a $2 \mathrm{D}$ ultrasound since the first trimester of pregnancy. The suspect of conjoined twins comes from the inability to see a clear separation between the fetal anatomic segments.

The diagnosis however should be done after an accurate ultrasound evaluation combined with the evaluation of the fetal movements. An accurate anatomic and vascular map is fundamental for the evaluation of the joined organ and to determine the prognosis.

Due to the high frequency of associated anomalies related to fetal fusion, including neural tube defect, orofacial cleft, imperforate anus and diaphragmatic hernia, a detailed ultrasound evaluation is still useful. For this aspect transvaginal 3D ultrasound offers an improvement on the conventional 2D ultrasound. This technique provides accurate data regarding the fetal biometry and structures using the multiplanar imaging as well as the surface rendering which can contribute to improve the diagnosis of fetal malformation.

Bega et al have reported that combining multiplanar display and surface rendering they were able to confirm a conjoined twin diagnosis at the 10th week of gestation and to assure the level and extention of the organ connection. ${ }^{30}$

The importance of 3D ultrasound for early diagnosis of cephalopagus twins has been reported by Kuroda and colleagues. ${ }^{32}$ We cannot assume that the $3 \mathrm{D}$ technique is decisive in conjoined twin diagnosis, but we can suggest that 3D ultrasound can be of great help in classifying more accurately the type of conjoined twins found. ${ }^{31-34}$

As stated by Bonila-Musoles et $\mathrm{al}^{34}$ the use of Color Doppler combined with 3D ultrasound can be a useful addition to 2D technique when confirming an early diagnosis and determining the extention of the conjoined organ and the ultimate classification of conjoined twins. 


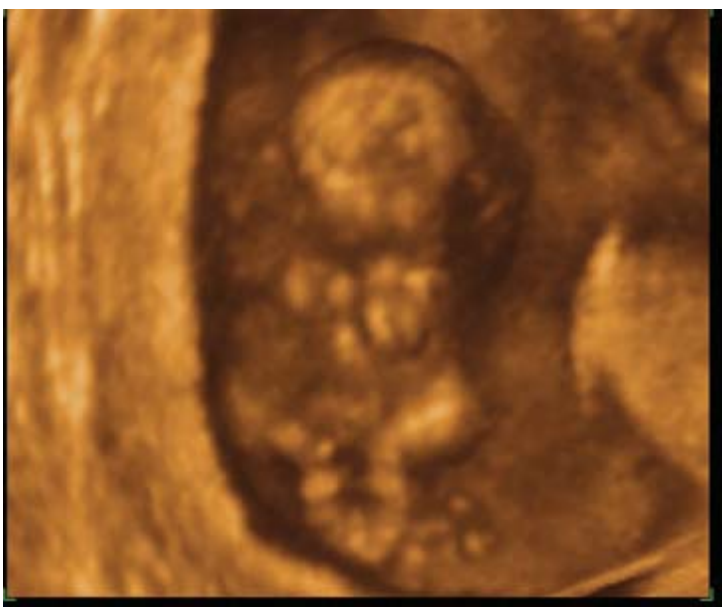

Fig. 3: A case of thoraco-omphalopagus conjoined twins at 12 weeks of gestation by surface rendering mode

Some authors have reported that 3D and 4D techniques improve prenatal assessment of conjoined twins with particular attention to vascular intertwin connections ${ }^{35}$ (Figs 3 and 4).

\section{D ULTRASOUND}

Various studies have been carried out with the conventional 2D technique on evaluating the fetal motorial pattern as early as the first trimester allowing an outline of several motorial patterns during pregnancy, expressing the evolution of the maturative process of the central nervous system during intra uterine life.

We have so seen a progressive development of fetal activity which starts with cardiac activity (7th week), it is then followed by torso movements (9th week), and the first limb movements (9th week). Between the 9th and the 12th week we notice rapid changes of position and posture. Subsequently we see isolated or armonic flexion-extention movements of the whole body at 12 to 13 weeks. ${ }^{36}$

Several studies have followed on the evaluation of fetal motorial and behavioral patterns with the support of 4DUS. This field gives us the possibility to better define the degree of normality and pathology of fetal neurologic functionality in uterus so to asses in prenatal time which fetuses can be at risk of neurologic outcome independently of intrapartum management. ${ }^{37-39}$

\section{CONCLUSIONS}

Some authors showed that with 3DUS we can better identify anatomic structures than with the bidimentional technique, allowing a more accurate evaluation of the fetus anatomy, particularly in those cases where an increase of chromosomic anomaly risk is present. ${ }^{40}$

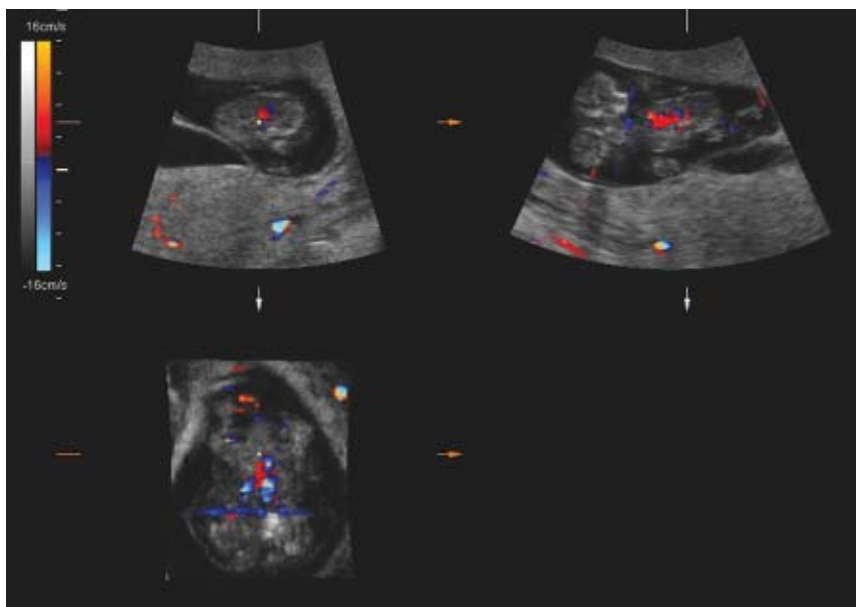

Fig. 4: 3D multiplanar color Doppler shows the communication of the vessels between the thoraco-omphalopagus conjoined twins

The main advantages are obtained when working on the central nervous system (CNS), with superficial anomalies (e.g. facial anomalies), anomalies of the skeleton and in particular of the limbs. ${ }^{21,41-43}$ The disadvantages of the 3D technique are mainly linked to the characteristics of maternal tissue, the reduction of amniotic fluid and fetal or maternal movements which interfere with a good acquisition and storage of 3D images.

At present, the comparison of this new technique with the conventional one has not been extensive. Nevertheless 3D imaging is finding a role in studies of embryo-fetal morphology, not only in normal cases, but especially in pathologies where the potential of this technology is more interesting. The common opinion at the moment is that 3D ultrasound is not a substitution for the conventional 2D ultrasound, but it is seen as an increasingly useful complementary technique and integration to the conventional ultrasound approach.

\section{REFERENCES}

1. Nelson TR, Downey DB, Pretorius DH, Fenster A. Threedimensional ultrasound. Philadelphia: Lippincott, Williams \& Wilkins, 1999.

2. Baba K, Satoh K, Sakamoto S, Okai T, Ishii S. Development of an ultrasonic system for three-dimensional reconstruction of the fetus. J Perinat Med 1989;17:19-24.

3. Baba K, Jurkovic D. Three-dimensional ultrasound in obstetrics and gynecology. New York: Parthenon Publishing Group, 1997.

4. Merz E, Bahlmann F, Weber G. Volume scanning in the evaluation of fetal malformations: a new dimension in prenatal diagnosis. Ultrasound Obstet Gynecol 1995;5:222-7.

5. Platt L, Santulli T, Carlson DE, Greene N, Walla CA. Threedimensional ultrasonography in obstetrics and gynecology: preliminary experience. Am J Obstet Gynecol 1998;178(6): 1199-1206. 
6. Dyson RL, Pretorius DH, Budorick NE, Johnson DD, Sklansky MS, Cantrell CJ, Lai S, Nelson TR. Three-dimensional ultrasound in the evaluation of fetal anomalies. Ultrasound Obstet Gynecol 2000;16:321-8.

7. Baba K, Okai T. Basis and principles of three dimensional ultrasound. In Baba K, Jurkovic D (Eds): Three-dimensional Ultrasound in Obstetrics and Gynecology. Carnforth, UK: Parthenon Publishing, 1997;1-20.

8. Brandl H, Gritzky A, Hainzinger M. 3D ultrasound: a dedicated system. Eur Radiol 1999;9(Suppl 3):331-3.

9. Merz E, Weber G, Bahlmann F, Miric-Tesanic D. Application of transvaginal and abdominal three-dimensional ultrasound for the detection or exclusion of fetal malformations of the fetal face. Ultrasound Obstet Gynecol 1997;9:237-43.

10. Lee A, Deutinger J, Bernaschek G. Three dimensional ultrasound: abnormalities of the fetal face in surface and volume rendering mode. Br J Obstet Gynecol 1995;102(4):302-6.

11. Sleurs E, Gonçalves LF, Johnson A, Espinoza J, Devers P, Chaiworapongsa T, Romero R. First-trimester ultrasonographic findings in a fetus with frontonasal malformation. The Journal of Maternal-Fetal and Neonatal Medicine 2004;16:187-97.

12. Takeuchi H. First-trimester diagnosis of fetal abnormalities by two- and three-dimensional sonoembriology. In Kurjak A, Chervenak A (Eds): The Fetus as a Patient. Parthenon Publishing, 2002;52- 65 .

13. Takeuchi H. Sonoembryology in the central nervous system. In Kurjak A, Chervenak A (Eds): The Fetus as a Patient. Carnforth, UK: Parthenon Publishing 1994;141-50.

14. Blaas H-G, Eik-Nes SH, Kiserud T, Hellevik LR. Early development of the forebrain and midbrain: a longitudinal ultrasound study from 7 to 12 weeks of gestation. Ultrasound Obstet Gynecol 1994;4:183-92.

15. Blaas H-G, Eik-Nes SH, Kiserud T, Hellevik LR. Early development of the hindbrain: a longitudinal ultrasound study from 7 to 12 weeks of gestation. Ultrasound Obstet Gynecol 1995;5:151-60.

16. Gonzales-Gomez F, Salamanca A, Padilla M C, Camara M, Sabatel RM. Alobar holoprosencephalic embryo detected via transvaginal sonography. Eur J Obstet Gynecol Reprod Biol 1992;47:266-70.

17. Blaas H-G, Eik-Nes SH, Vainio T, Isaksen CV. Alobar holoprosencephaly at 9 weeks gestational age visualized by twoand three-dimensional ultrasound. Ultrasound Obstet Gynecol 2000;15:62-5.

18. Chuang L, Chang C-H, Yu C-H, Chang FM. Three-dimensional sonographic visualization of a fetal omphalocele at 14 weeks of gestation. Prenat Diagn 2000;20:523-4.

19. Lee A, Kratochwil A, Deutinger J, Bernaschek G. Threedimensional ultrasound in diagnosing phocomelia. Ultrasound Obstet Gynecol 1995;5(4):238-40.

20. Van Keirsbilck J, Cannie M, Robrechts C, de Ravel T, Dymarkowski S, Van den Bosch T, Van Schoubroeck D. First trimester diagnosis of sirenomelia. Prenat Diagn 2006;26:684-8.

21. Hata T, Aoki S, Akiyama M, Yanagihara T, Miyazaki K. Threedimensional ultrasonographic assessment of fetal hands and feet. Ultrasound Obstet Gynecol 1998;12(4):235-9.
22. Snijders RJM, Noble P, Sebire N, Souka N, Nicolaides KH. UK multicentre project on assessment of risk of trisomy 21 by maternal age and fetal nuchal translucency thickness at 10-14 weeks of gestation. Lancet 1998;351:343-6.

23. Kurjak A, Kupesic S, Kosuta-Ivanevic M. Three-dimensional transvaginal ultrasound improves measurement of nuchal translucency. J Perinat Med 1999;27:97-102.

24. Eppel W, Christof W, Frigo P, Lee A. Three-versus twodimensional ultrasound for nuchal translucency thickness measurements: comparison of feasibility and levels of agreement. Prenat Diagn 2001;21:596-601.

25. Cicero S, Curcio P, Papageorghiou A, Sonek J, Nicolaides KH. Absence of nasal bone in fetus with trisomy 21 at 11-14 weeks of gestation: an observational study. Lancet 2001;358:1665-7.

26. Zoppi MA, Ibba RM, Axiana C, Floris M, Manca F, Monni G. Absence of fetal nasal bone and aneuploidies at first trimester nuchal tranalucency screening in unselected pregnancies. Prenat Diagn 2003;23:496-500.

27. Rembouskos G, Cicero S, Longo D, Vandecruys H, Nicolaides $\mathrm{KH}$. Assessment of the fetal nasal bone at 11-14 weeks of gestation by three-dimensional ultrasound. Ultrasound Obstet Gynecol 2004;23:232-6.

28. Peralta CFA, Falcon O, Wegrzyn P, Faro C, Nicolaides KH. Assessment of the gap between the fetal nasal bones at 11 to 13 +6 weeks of gestation by three-dimensional ultrasound. Ultrasound Obstet Gynecol 2005;464-7.

29. Benoit B, Chaoui R. Three-dimensional ultrasound with maximal mode rendering: a novel technique for the diagnosis of bilateral or unilateral absence or hypoplasia of nasal bones in secondtrimester screening for Down syndrome. Ultrasound Obstet Gynecol 2005;25:19-24.

30. Bega G, Wapner R, Lev-Toaff A, Kuhlman K. Diagnosis of conjoined twins at 10 weeks using three-dimensional ultrasound: a case report. Ultrasound Obstet Gynecol 2000;388-90.

31. Maymon R, Halperin R, Weinraub Z, Herman H, Schneider D. Three-dimensional transvaginal sonography of conjoined twins at 10 weeks; a case report. Ultrasound Obstet Gynecol 1998;11:292-4.

32. Kuroda K, Kamei Y, Kozuma S, Kiluchi A, Fujii T, Unno N, Baba K, Taketani Y. Prenatal evaluation of cephalophagus conjoined twins by mens of three-dimensional ultrasound at 13 weeks of pregnancy. Ultrasound Obstet Gynecol 2000;16:2646.

33. Jonson D, Pretorius E, Budorick N. Three-dimensional ultrasound of conjoined twins. Obstet Gynecol 1997;90:701-2.

34. Bonilla-Musoles F, Raga F, Bonilla F Jr, Blanes J, Osborne N. Early diagnosis of conjoined twins using two-dimensional color Doppler and three-dimensional ultrasound. J Nat Med Assoc 1998;90:552-6.

35. Allegra A, Monni G, Zoppi MA, Curcio P, Marino A, Volpe A: Conjoined twins in a trichorionic quadruplet pregnancy after intracytoplasmic sperm injection and quarter laser assisted zona thinning. Fertil Steril 2007;87:189.e9-189.e12.

36. Reinold E. Identification and differentiation of fetal movements. Contrib Gynecol Obstet 1979; 6: 29:32. 
37. Kurjak A, Stanojevit M, Andonotopo W, Scazzocchio-Duenas, Azumendi G, Carrera JM. Fetal behavior assessed in all three trimesters of normal pregnancy by four-dimensional ultrasonography. Croat Med J 2005;46(5):772-80.

38. Campbell S. 4D, or not 4D: that is the question. Ultrasound Obstet Gynecol 2002;19:1-3.

39. Boue J, Vignal P, Aubrey JP, Aubrey MC, Aleese JM. Ultrasound movement patterns of fetuses with chromosomal anomalies. Prenat Diagn 1982;2: 61-5.
40. Benoit B, Halfner T, Kuriak A, Kupesic S, Bekavac I, Bozek T. Three-dimensional sonoembryology. J Perinatal Med 2002:30(1):63-73. Review.

41. Timor-Tritsch IE, Platt LD. Three-dimensional ultrasound experience in obstetrics. Curr Opin Obstet Gynecol 2002;14(6):569-75.

42. Shih JC, Jaffe R, Hsieh FJ. Three-dimensional ultrasonography in early pregnancy. Semin perinatol. 2001;25(1):3-10.

43. Hull AD, James G, Salerno C, Nelson T, Pretorius D. Threedimensional ultrasound and assessment of the first trimester fetus. J Ultrasound Med 2001;20(4):287-93. 